PROCEEDINGS OF THE

AMERICAN MATHEMATICAL SOCIETY

Volume 132, Number 9, Pages 2783-2792

S 0002-9939(04)07408-8

Article electronically published on March 25, 2004

\title{
FIRST COUNTABLE EXTENSIONS OF REGULAR SPACES
}

\author{
PETR SIMON AND GINO TIRONI \\ (Communicated by Alan Dow)
}

\begin{abstract}
We investigate feebly compact extensions of first countable regular locally feebly compact spaces. Solutions of problems posed by R. M. Stephenson, Jr. and G. M. Reed are given.
\end{abstract}

\section{Introduction}

Recall a few notions. A space $X$ is feebly compact if every locally finite collection of nonempty open sets is finite. A space $X$ is pseudocompact if it is completely regular and every real-valued continuous function defined on $X$ is bounded. It is well known that feeble compactness and pseudocompactness differ in the class of regular spaces and coincide in the class of Tychonoff spaces. An extension of a space $X$ is a space $Y$ containing (a homeomorphic copy of) the space $X$ as a dense subspace.

Our research was motivated by a problem posed by R. M. Stephenson, Jr.:

Does every locally feebly compact, first countable regular space have a feebly compact first countable regular extension? [S, Question 23].

There are a vast number of problems, the nature of which is closely related to the just mentioned sample. A good survey on this subject may be found in the introductory part of S. Watson's paper [W].

The main goal of the present paper is to introduce a technique that allows us to deal with problems of this sort. Then we show that

Every locally feebly compact regular space $X$ can be embedded as a dense open subspace in a feebly compact regular space $Y$ that is first countable at every point from $Y \backslash X$.

Every separable, locally feebly compact Moore space embeds as an open dense set in a feebly compact Moore space.

Every locally pseudocompact, separable Moore space embeds as an open dense set in a pseudocompact Moore space.

There is a Moore connected pseudocompact space without a dense relatively countably compact subset.

Received by the editors October 3, 2002 and, in revised form, May 26, 2003.

2000 Mathematics Subject Classification. Primary 54D35; Secondary 54E30, 54D25, 54D80.

Key words and phrases. Feebly compact space, Moore space, first countable space, extension.

The authors gratefully acknowledge support provided by grants GAČR 201/00/1467 and GAUK 270/2001 (P. Simon) and by research funds of the University of Trieste (ex 60\%) and of the "Real Analysis" PRIN group of MIUR (G. Tironi). 
The first two theorems answer Stephenson's questions 23 and 25 from [S] and significantly strengthen [T, Theorem 2.2]. The third theorem answers Reed's questions 4.(7) from [R2 and 5.2 from [R3]. This theorem was announced by P. Nyikos in 1991. During the long subsequent period of eleven years, he has not published any paper with a proof of it. Therefore we believe that our third theorem is also new. The last theorem improves S. Watson's result $\mathrm{W}$, Theorem 3.1] in three directions. "First countable" is replaced by "Moore", $\mathrm{CH}$ is replaced by ZFC, and the complete regularity is proved.

The research resulting in this paper was done when the first named author was a guest of the Department of Mathematics at the University of Trieste, with welcome support provided by G.N.A.M.P.A. of I.N.d.A.M. He wishes to express his sincere thanks to all members of the department for their hospitality.

\section{General setting}

The notation used in this paper follows the common standard. We use the topological terminology from Engelking's book [E]. That in particular means that regular spaces are always $\mathrm{T}_{1}$. If $X$ is a topological space, $\operatorname{Open}^{+}(X)$ denotes the set of all nonempty open subsets of $X$.

If $\phi$ is a property of topological spaces, we shall say that a space $X$ is locally $\phi$, if every point has a neighborhood having $\phi$. If $M \subseteq X$, then we say that $M$ is relatively $\phi$, if the subspace $\bar{M}$ is $\phi$.

As we can see later, whenever $Y$ is an extension of $X$, then the points of $Y \backslash X$ will belong to $\mathcal{P} \mathcal{P} \mathcal{P}(X)$, despite the fact that $\mathcal{P P}(X)$ suffices. The only reason for that is notational convenience.

Throughout the rest of the paper, all spaces are assumed to be regular.

Suppose $X$ is a regular space that is not feebly compact. Denote by $\mathcal{D}(X)$ a set of some countably infinite discrete collections of nonempty open subsets of $X$, in symbols, $\mathcal{D}(X) \subseteq\left\{G \in\left[\operatorname{Open}^{+}(X)\right]^{\omega}: G\right.$ is discrete $\}$.

We shall introduce five relations on $\mathcal{D}(X)$.

- For $F, G \in \mathcal{D}(X)$, we shall say that $F$ is essentially contained in $G$, and write $F \subseteq \subseteq^{\text {ess }} G$, if there are a cofinite $F^{\prime} \subseteq F$ and an injection $\varphi: F^{\prime} \longrightarrow G$ satisfying $\left(\forall U \in F^{\prime}\right) U \subseteq \varphi(U)$.

- For $F, G \in \mathcal{D}(X)$, we shall say that $F$ is strictly essentially contained in $G$, and write $F \subset^{\text {ess }} G$, if there are a cofinite $F^{\prime} \subseteq F$ and an injection $\varphi: F^{\prime} \longrightarrow G$ satisfying $\left(\forall U \in F^{\prime}\right) \bar{U} \subseteq \varphi(U)$.

- For $F, G \in \mathcal{D}(X)$, we shall say that $F$ is essentially equal to $G$, in symbols $F={ }^{\text {ess }} G$, if the symmetric difference $(F \backslash G) \cup(G \backslash F)$ is finite.

- For $F, G \in \mathcal{D}(X)$, we shall say that $F$ is essentially disjoint from $G$, and write $F \perp G$, if the set $\{U \in F: U \cap \bigcup G \neq \emptyset\}$ is finite.

- For $F, G \in \mathcal{D}(X)$, we shall say that $F$ is essentially compatible with $G$, and write $F \| G$, if $F \perp G$ does not hold.

Our first statement summarizes trivialities, and its proof is left to the reader.

\section{Observation 1.1.}

(i) Both $\subseteq^{\text {ess }}$ and $\subset^{\text {ess }}$ are transitive and reflexive relations on $\mathcal{D}(X)$.

(ii) If $F, G \in \mathcal{D}(X)$ and $F \subset \subset^{\text {ess }} G$, then $F \subseteq{ }^{\text {ess }} G$.

(iii) If $F, G \in \mathcal{D}(X)$ are such that $F \subseteq{ }^{\text {ess }} G$ and $G \subseteq \subseteq^{e s s} F$, then $F={ }^{\text {ess }} G$.

(iv) If $F, G \in \mathcal{D}(X)$ are such that $F \subset{ }^{e s s} G$ and $G \subset{ }^{\text {ess }} F$, then $F={ }^{\text {ess }} G$ and all sets in $F \cap G$ are clopen. 
Recall that a filter on a quasi-order $(P, \leq)$ is a family $f \subseteq P$ satisfying the following:

- $f \neq \emptyset$;

- if $F \in f, G \in P$ and $F \leq G$, then $G \in f$;

- if $F, G \in f$, then there is some $H \in f$ with $H \leq F, H \leq G$.

A filter $f$ is called an ultrafilter, if no proper $g \supset f$ is a filter 1 Denote by $\operatorname{Ult}(P, \leq)$ the family of all ultrafilters on $(P, \leq)$.

Example 1. Let $X$ be the discrete space $\omega$, and let $\mathcal{A}$ be a maximal almost disjoint family on $\omega$. Let $\mathcal{D}(X)$ be the collection $\{F(A): A \in \mathcal{A}\}$, where $F(A)=$ $\{\{n\}: n \in A\}$. Then $\left(\mathcal{D}(X), \subseteq{ }^{\text {ess }}\right)$ is a discrete quasiorder, i.e., no two members are comparable. Therefore every ultrafilter on $\left(\mathcal{D}(X), \subseteq^{\text {ess }}\right)$ is just a singleton set $\{F(A)\}$ for some $A \in \mathcal{A}$, and $\operatorname{Ult}(\mathcal{D}(X))=\{\{F(A)\}: A \in \mathcal{A}\}$.

Definition 1.2. Suppose $X$ is a regular space and $\mathcal{D}(X)$ is as above. Let us define a space $\Psi\left(\mathcal{D}(X), \subseteq^{\text {ess }}\right)$ as follows. The underlying set is a disjoint union of $X$ and $\operatorname{Ult}\left(\mathcal{D}(X), \subseteq^{\text {ess }}\right)$. The topology is described by means of neighborhood bases:

- $X$ is an open subspace of $\Psi\left(\mathcal{D}(X), \subseteq^{\text {ess }}\right)$.

For $F \in \mathcal{D}(X)$, denote by $\widehat{F}$ the set $\{f \in \operatorname{Ult}(\mathcal{D}(X), \subseteq$ ess $): F \in f\}$.

- For $f \in \operatorname{Ult}\left(\mathcal{D}(X), \subseteq^{\text {ess }}\right), F \in f$ and $K \in[F]^{<\omega}$, let $O(f, F, K)$ be the set $\widehat{F} \cup \bigcup F \backslash \bigcup K$. The family $\left\{O(f, F, K): F \in f, K \in[F]^{<\omega}\right\}$ is a local base at $f$.

At this moment, we cannot say anything interesting about $\Psi\left(\mathcal{D}(X), \subseteq \subseteq^{\text {ess }}\right)$ except for two vital facts.

Lemma 1.3. $\Psi\left(\mathcal{D}(X), \subseteq \subseteq^{\text {ess }}\right)$ is a topological space.

Proof. Any set open in $X$ is open also in $\Psi\left(\mathcal{D}(X), \subseteq{ }^{\text {ess }}\right)$. Let $f \in \operatorname{Ult}\left(\mathcal{D}(X), \subseteq^{\text {ess }}\right)$ and consider the set $O(f, F, K)$. If $x \in X, x \in O(f, F, K)$, then $x \in \bigcup F \backslash \bigcup K$, which is an open neighborhood of $x$ in $X$ and consequently also in $\Psi\left(\mathcal{D}(X), \subseteq{ }^{\text {ess }}\right)$. If $f^{\prime} \in O(f, F, K)$, then $F \in f^{\prime}$ and so $O\left(f^{\prime}, F, K\right)=O(f, F, K)$. So the neighborhood $O(f, F, K)$ of a point $f \in \operatorname{Ult}\left(\mathcal{D}(X), \subseteq \subseteq^{\text {ess }}\right)$ is already open in $\Psi\left(\mathcal{D}(X), \subseteq^{\text {ess }}\right)$.

Lemma 1.4. If $f \in \Psi\left(\mathcal{D}(X), \subseteq \subseteq^{e s s}\right) \backslash X$ and $x \in X$, then $x$ and $f$ have disjoint open neighborhoods.

Proof. Let $F \in \mathcal{D}(X)$ be such that $f \in \widehat{F}$. The family $F$ is discrete in $X$. So there is an open neighborhood $W$ of $x$ for which there is at most one member, say $U \in F$, with $W \cap U$ nonempty. The sets $W$ and $O(f, F,\{U\})$ are disjoint.

Consider $X$ and $\mathcal{D}(X)$ from Example 1. The reader should immediately see that in this special case, the space $\Psi\left(\mathcal{D}(X), \subseteq{ }^{\text {ess }}\right)$ is nothing else than the familiar space $\Psi(\mathcal{A})$ from [GJ, Ex. 5I]. This explains and perhaps even justifies our notation.

From now on, we will gradually impose some restrictions on the general situation in order to be able to prove more. Also, we will write simply $\mathcal{D}(X)$ instead of $\left(\mathcal{D}(X), \subseteq^{\mathrm{ess}}\right)$.

Lemma 1.5. Suppose $\mathcal{D}(X)$ is such that for distinct $f, g \in \operatorname{Ult}(\mathcal{D}(X))$ there are $F \in f$ and $G \in g$ such that either $F \perp G$ or $G \perp F$. Then the space $\Psi(\mathcal{D}(X))$ is Hausdorff.

\footnotetext{
${ }^{1}$ Notice that in the special case when $(P, \geq)$ is a tree, filters coincide with chains and ultrafilters with branches. This will be the case in the next paragraph.
} 
Proof. Consider $f \neq g$, both belonging to $\Psi(\mathcal{D}(X)) \backslash X$. Choose $F \in f$ and $G \in g$ so that $F \perp G$. Then $\{U \in F: U \cap \bigcup G \neq \emptyset\}$ is a finite subset of $F$; denote it by $K$. Now it is clear that $O(f, F, K)$ and $O(g, G, \emptyset)$ are disjoint neighborhoods separating $f$ from $g$. This, together with Lemma 1.4 and the fact that $X$ is regular, shows that $\Psi(\mathcal{D}(X))$ is Hausdorff, and similarly if $G \perp F$.

The relations $\perp$ and $\|$ are not, in general, symmetric. However, a careful choice of $\mathcal{D}(X)$ may help, as shown below. We omit the easy proof.

Lemma 1.6. Suppose that every member of $\mathcal{D}(X)$ consists of relatively feebly compact open sets. Then $\perp$ and $\|$ are symmetric relations.

Our next observations concern regularity.

Lemma 1.7. Let $V$ be a relatively feebly compact neighborhood of a point $x \in X$. Then there is a neighborhood $W$ of $x$ such that $\bar{W} \subseteq V$ in $\Psi(\mathcal{D}(X))$.

Proof. The space $X$ is regular. So there is a neighborhood $W$ of $x$ such that $\bar{W} \subseteq V$. It is enough to show that $\bar{W}$ is a closed set also in $\Psi(\mathcal{D}(X))$. Pick an arbitrary $f \in \Psi(\mathcal{D}(X)) \backslash X$ and an arbitrary $F \in f$. Then the set $K=\{U \in F: U \cap V \neq \emptyset\}$ is finite, since $V$ is relatively feebly compact and $F$ is discrete. Consequently $O(f, F, K)$ is a neighborhood of $f$ disjoint with $\bar{W}$.

Example 2. The assumption that $X$ is locally feebly compact at $x$ is essential. To see this, consider a standard example, the Fréchet-Urysohn fan $S_{\omega}$, the quotient space of $(\omega+1) \times \omega$ with all points $\langle\omega, n\rangle$ identified to a single point $\infty$. Let $\mathcal{D}\left(S_{\omega}\right)$ consist of all discrete families that have their unions closed in $S_{\omega}$. Then the closed set $\Psi\left(\mathcal{D}\left(S_{\omega}\right)\right) \backslash S_{\omega}$ and the point $\infty$ cannot be separated.

Lemma 1.8. Suppose $\mathcal{D}(X)$ has the following two properties:

(a) for every $f \in \operatorname{Ult}(\mathcal{D}(X)$ ) and every $F \in f$ there is some $G \in f$ with $G \subset \subset^{e s s} F$;

(b) for every $G \in \mathcal{D}(X), \widehat{G} \cup \bigcup\{\widehat{H}: H \in \mathcal{D}(X), H \perp G\}=\operatorname{Ult}(\mathcal{D}(X))$.

Then the space $\Psi(\mathcal{D}(X))$ is regular at each $f \notin X$.

Proof. Let $f \in \operatorname{Ult}(\mathcal{D}(X)), F \in f$ and a finite $K \subseteq F$ be arbitrary. Choose $G \in f$ such that $G \subset \subset^{\text {ess }} F$; this is possible by (a). We have a cofinite $G^{\prime} \subseteq G$ and an injection $\varphi: G^{\prime} \longrightarrow F$ with $\bar{V} \subseteq \varphi(V)$ whenever $V \in G^{\prime}$. Let $G^{\prime \prime}=\bar{G}^{\prime} \backslash \varphi^{-1}[K]$. Since $G^{\prime}$ is cofinite, $K$ finite and $\varphi$ an injection, the set $G^{\prime \prime}$ is a cofinite subset of $G$ as well. By the choice of $G^{\prime \prime}$, we have $O\left(f, G, G \backslash G^{\prime \prime}\right) \subseteq O(f, F, K)$.

Suppose that a point $x \in X$ belongs to $\bigcup G^{\prime \prime}$. The family $G$ is discrete. So there is a unique $V \in G^{\prime \prime}$ with $x \in \bar{V}$. Having $\bar{V} \subseteq \varphi(V) \in F \backslash K$, we have $x \in O(f, F, K)$.

Suppose that a point $f^{\prime} \in \operatorname{Ult}(\mathcal{D}(X))$ belongs to $\overline{O\left(f, G, G \backslash G^{\prime \prime}\right)}$. Then (b) implies that $G \in f^{\prime}$. So $O\left(f^{\prime}, G, G \backslash G^{\prime \prime}\right)$ is a neighborhood of $f^{\prime}$ and then $f^{\prime} \in O(f, F, K)$. The proof is complete.

\section{Feebly compact extensions}

Stephenson's question will be answered in Theorem 2.3 below. But first we prove a lemma that will again reduce the general situation from the previous section to a yet more special case. 
Definition 2.1. Let $X$ be a topological space, and let $\mathcal{G} \subseteq \mathcal{P} \mathcal{P}(X)$ be a family such that each member of $\mathcal{G}$ is a countably infinite discrete collection consisting of open nonvoid sets. We shall say that $\mathcal{G}$ is dense if the following holds: For each discrete $F \in\left[\operatorname{Open}^{+}(X)\right]^{\omega}$ there is some $G \in \mathcal{G}$ with $G \subseteq \subseteq^{\text {ess }} F$.

Lemma 2.2. Suppose that $X$ is a locally feebly compact regular space, and that $\mathcal{G}$ is a dense collection consisting of infinite discrete families with open relatively feebly compact elements. Then for each maximal essentially disjoint family $\mathcal{A} \subseteq \mathcal{G}$ there exists a maximal essentially disjoint family $\mathcal{B} \subseteq \mathcal{G}$ such that for every $A \in \mathcal{A}$ and $B \in \mathcal{B}$, either $A \perp B$ or $B \subset^{\text {ess }} A$.

Proof. For each $A \in \mathcal{A}$, let $\mathcal{B}(A)$ be a family that is maximal with respect to

(i) $\mathcal{B}(A) \subseteq \mathcal{G}$;

(ii) if $B \in \mathcal{B}(A)$, then $B \subset^{\text {ess }} A$;

(iii) if $B, B^{\prime} \in \mathcal{B}(A)$ are distinct, then $B \perp B^{\prime}$.

Put $\mathcal{B}=\bigcup\{\mathcal{B}(A): A \in \mathcal{A}\}$. Then $\mathcal{B}$ is as required: Obviously $\mathcal{B} \subseteq \mathcal{G}$, and if $A \in \mathcal{A}$ and $B \in \mathcal{B}$, then either $B \in \mathcal{B}(A)$, in which case $B \subset \subset^{\text {ess }} A$, or $B \in \mathcal{B}\left(A^{\prime}\right)$ for some $A^{\prime} \in \mathcal{A}, A^{\prime} \neq A$. But then $A \perp A^{\prime}$ and $B \subset^{\text {ess }} A^{\prime}$; hence $B \perp A$. This observation together with (iii) gives that the family $\mathcal{B}$ is essentially disjoint.

It remains to prove the maximality of $\mathcal{B}$. Let $F$ be an arbitrary infinite discrete family of open sets. Since $X$ is locally feebly compact, we may assume that $F \in \mathcal{G}$. By the maximality of $\mathcal{A}$, there is some $A \in \mathcal{A}$ with $F \| A$. Since both $A$ and $F$ consist of open relatively feebly compact sets and $F \| A$, an easy induction will produce an infinite subset $F^{\prime} \subseteq F$ and an injection $\varphi: F^{\prime} \longrightarrow A$ such that for each $U \in F^{\prime}, U \cap \varphi(U) \neq \emptyset$. For every $U$ in $F^{\prime}$ choose an open set $V_{U}$ satisfying $\overline{V_{U}} \subseteq U \cap \varphi(U)$. For the family $H=\left\{V_{U}: U \in F^{\prime}\right\}$ we have $H \in \mathcal{G}$ and $H \subset^{\text {ess }} A$. Since $\mathcal{B}(A)$ is a maximal family satisfying (i), (ii) and (iii), there must be some $B \in \mathcal{B}(A)$ with $B \| H$. For this $B$ we also have $B \| F$, which shows the maximality of $\mathcal{B}$ and concludes the proof of the lemma.

Theorem 2.3. Every locally feebly compact regular space $X$ can be embedded as a dense open subspace in a feebly compact regular space $Y$ that is first countable at every point from $Y \backslash X$.

Proof. Denote by $\mathcal{G}$ the collection of all countably infinite discrete families consisting of open relatively feebly compact sets. The family $\mathcal{G}$ is dense, because $X$ is locally feebly compact.

Let $\mathcal{A}_{0}$ be an arbitrary maximal essentially disjoint subfamily of $\mathcal{G}$. Proceed by an induction to $\omega$. Once the family $\mathcal{A}_{n}$ is known, then $\mathcal{A}_{n+1}$ will be the result of an application of Lemma 2.2 to $\mathcal{A}_{n}$.

Put $\mathcal{D}(X)=\bigcup\left\{\mathcal{A}_{n}: n \in \omega\right\}$ and $Y=\Psi(\mathcal{D}(X))$. Observe that $\mathcal{D}(X)$ has a very simple structure: Whenever $F, G$ are distinct members of $\mathcal{D}(X)$, then either $F \perp G$ or $F \subset \subset^{\text {ess }} G$ or $G \subset \subset^{\text {ess }} F$. So every ultrafilter on $\mathcal{D}(X)$ is just an $\subset{ }^{\text {ess }}$-decreasing collection $\left\{F_{n}: n \in \omega\right\}$ with $F_{n} \in \mathcal{A}_{n}$.

The space $X$ is an open subspace of $Y$ by the definition of $\Psi(\mathcal{D}(X))$, and the space $Y$ is Hausdorff by 1.5 and regular by 1.7 and 1.8 (to check (b) from 1.8, observe that $\bigcup\left\{\widehat{F}: F \in \mathcal{A}_{n}\right\}=\operatorname{Ult}(\mathcal{D}(X))$ for every $\left.n \in \omega\right)$.

The space $Y$ is feebly compact. Suppose not: let $F$ be an infinite discrete collection of open subsets of $Y$. Since $X$ is a dense open subset of $Y$ and since $\mathcal{G}$ is dense, we may and shall assume that $F \in \mathcal{G}$. By the maximality of $\mathcal{A}_{0}$, there is 
some $A_{0} \in \mathcal{A}_{0}$ such that $A_{0}$ and $F$ are not essentially disjoint. Arguing exactly the same way as in the proof of Lemma 2.2, we can find $A_{1} \in \mathcal{A}_{1}, A_{1} \| F$. Proceeding further, we get a sequence $g=\left\{A_{n}: n \in \omega\right\}$ such that for each $n \in \omega$ we have $A_{n} \in \mathcal{A}_{n}, A_{n+1} \subset{ }^{\text {ess }} A_{n}$, and $A_{n} \| F$. We already know that such a $g$ belongs to $\operatorname{Ult}(\mathcal{D}(X))$. The point $g$ clearly witnesses that the family $F$ is not discrete, a contradiction.

The space $Y$ is first countable in each point from $Y \backslash X$ : Whenever $f \in$ $\operatorname{Ult}(\mathcal{D}(X)), f=\left\{F_{n}: n \in \omega\right\}$, then the open local basis $\left\{O\left(f, F_{n}, K\right): n \in \omega\right.$, $\left.K \in\left[F_{n}\right]^{<\omega}\right\}$ is obviously countable.

The proof of Theorem 2.3 will serve as a model proof for the next three theorems. We will only pinpoint the modifications necessary for the case in question. For instance, we shall show now how to preserve the property of being Moore.

Theorem 2.4. Every separable, locally feebly compact Moore space embeds as an open dense set in a feebly compact Moore space.

Proof. Denote by $\left\{q_{n}: n \in \omega\right\}$ some countable dense subset of $X$. For each $n \in \omega$, choose an open relatively feebly compact neighborhood $W_{n}$ of a point $q_{n}$. Put $W=\bigcup_{n \in \omega} W_{n}$ and $Z=X \backslash W$.

The only modification of the proof of 2.3 will be a minor change of the starting family $\mathcal{G}$. Let $\mathcal{G}$ consist of all infinite discrete families with open relatively feebly compact elements, such that whenever $F \in \mathcal{G}$, then $\bigcup F \subseteq W$. Since $W$ is open dense in $X$, the family $\mathcal{G}$ is dense. Continue as in Theorem 2.3 then.

To see that $Y$ is a Moore space, choose a development $\Omega=\left\{\mathcal{O}_{n}: n \in \omega\right\}$ for $X$. Our task is to extend it into a development for $Y$.

Let $n \in \omega$. Every set $W_{j}$ is relatively feebly compact. So for every $F \in \mathcal{D}(X)$ one must find a finite set $F_{n} \subseteq F$ such that $\bigcup F \backslash \bigcup F_{n}$ is disjoint with $\bigcup_{j \leq n} W_{j}$. Put

$$
\mathcal{P}_{n}=\mathcal{O}_{n} \cup\left\{\bigcap\left\{O\left(f, F, F_{n}\right): F \in f \cap \mathcal{A}_{i}, i \leq n\right\}: f \in \operatorname{Ult}(\mathcal{D}(X))\right\} .
$$

We need to verify that $\left\{\mathcal{P}_{n}: n \in \omega\right\}$ is a development for $Y$. Let $y \in Y$, and let $O$ be an open neighborhood of $y$. We need to consider three cases:

- $y \in Z$ : Whenever $F \in \mathcal{G}$, then $\bigcup F \cap Z=\emptyset$. Consequently, no $O(f, F, K)$ for $f \in Y \backslash X$ contains $y$. Hence $s t\left(y, \mathcal{O}_{n}\right)=\operatorname{st}\left(y, \mathcal{P}_{n}\right)$. Therefore there is some $n$ with st $\left(y, \mathcal{P}_{n}\right) \subseteq O$, because $\Omega$ was a development for $X$.

- $y \in W$ : There is some $i \in \omega$ such that $y \in W_{i}$, and there is also some $k \in \omega$ such that $\operatorname{st}\left(y, \mathcal{O}_{k}\right) \subseteq O$. If $n$ is greater than the maximum of $i, k$, then $\operatorname{st}\left(y, \mathcal{O}_{n}\right) \subseteq O$, too, since $\mathcal{O}_{n} \subseteq \mathcal{O}_{k}$. However, if $F \in \mathcal{D}(X)$, then $\bigcup F \backslash \bigcup F_{i}$ is disjoint with $W_{i}$, and henceforth $y \notin O\left(f, F, F_{n}\right)$ for any $f \in \widehat{F}$. Thus $\operatorname{st}\left(y, \mathcal{P}_{n}\right)=\operatorname{st}\left(y, \mathcal{O}_{n}\right) \subseteq O$.

- $y \in Y \backslash X$ : For each $n \in \omega$, there is only one $F \in \mathcal{A}_{n}$ satisfying $F \in y$. Therefore $\operatorname{st}\left(y, \mathcal{P}_{n}\right)=\bigcap\left\{O\left(y, F, F_{n}\right): F \in y \cap \mathcal{A}_{i}, i \leq n\right\}$. There are some $H \in y$ and a finite set $H^{\prime} \subseteq H$ such that $y \in O\left(y, H, H^{\prime}\right) \subseteq O$. For every $U \in H^{\prime}$ pick some $q(U) \in Q$ with $q(U) \in U$. Since $H^{\prime}$ is finite, there is some $m \in \omega$ such that $\left\{q(U): U \in H^{\prime}\right\} \subseteq\left\{q_{j}: j \leq m\right\}$. There is a unique $k \in \omega$ such that $H \in \mathcal{A}_{k}$. If $n$ is bigger than both of $m, k$, then $H_{n} \supseteq H^{\prime}$. Now we have

$$
s t\left(y, \mathcal{P}_{n}\right)=\bigcap\left\{O\left(y, F, F_{n}\right): F \in y \cap A_{i}, i \leq n\right\} \subseteq O\left(y, H, H^{\prime}\right) \subseteq O,
$$

which completes the proof that $\left\{\mathcal{P}_{n}: n \in \omega\right\}$ is a development for $Y$. 
From Theorem 2.4, we immediately get

Corollary 2.5. Every separable, locally compact Moore space embeds as an open dense set in a feebly compact Moore space.

On the other hand, T. Terada and J. Terasawa proved

Theorem 2.6 (TT]). Every locally compact, first countable space embeds as a dense set in a pseudocompact first countable space.

There is no hope to see why and how to get Moore space directly from Theorem 2.6 , if the starting space was Moore. Therefore we must amalgamate complete regularity to our proof of Theorem 2.4, if we want to show

Theorem 2.7. Every locally pseudocompact separable Moore space embeds as an open dense set in a pseudocompact Moore space.

Proof. Let $W$ and $Z$ have the same meaning as in the proof of Theorem 2.4.

Let $\mathcal{G}$ consist of all countable discrete collections $G$ such that every $U \in G$ is a cozero set with a pseudocompact closure contained in $W$. Since $X$ is Tychonoff and locally pseudocompact, it is easy to see that $\mathcal{G}$ is dense.

Let $\mathcal{A}_{0}$ be an arbitrary maximal essentially disjoint subfamily of $\mathcal{G}$. Our effort now is to find $\mathcal{A}_{n}$ 's carefully enough to get a sufficiently rich family of continuous functions on the resulting $\Psi(\mathcal{D}(X))$. With the notation from the proof of Lemma 2.2 , we need to know how to pass from $A \in \mathcal{A}_{n}$ to $\mathcal{B}(A)$.

Let $F \in \mathcal{G}$, and let $\varphi: X \longrightarrow[0,1]$ be a continuous mapping. We shall say that $\varphi$ is a label for $F$, if for every $U \in F$ we have $\sup \{\varphi(x): x \in U\}=1$ and if for every $x \in X$ we have $\varphi(x)=0 \Longleftrightarrow x \notin \bigcup F$. Obviously, each $F \in \mathcal{G}$ has a label. Fix for every $F \subset \subset^{\text {ess }} A$ some label $\varphi_{F}$ and call the couple $\left\langle F, \varphi_{F}\right\rangle$ a labeled pair.

Let $\left\langle F, \varphi_{F}\right\rangle$ be a labeled pair and suppose that $G \subset \subset^{\text {ess }} F$. For a real $r \in[0,1]$, let us say that $\lim _{G} \varphi_{F}=r$ if

$$
\begin{aligned}
\sup \left\{\operatorname { i n f } \left\{\varphi_{F}(x): x\right.\right. & \left.\left.\in \bigcup G \backslash \bigcup G^{\prime}\right\}: G^{\prime} \in[G]^{<\omega}\right\} \\
& =\inf \left\{\sup \left\{\varphi_{F}(x): x \in \bigcup G \backslash \bigcup G^{\prime}\right\}: G^{\prime} \in[G]^{<\omega}\right\}=r .
\end{aligned}
$$

Observe that if $\left\langle F, \varphi_{F}\right\rangle$ is a labeled pair and $G \subseteq{ }^{\text {ess }} F$, then there is some $H \subset^{\text {ess }} G$ such that $\lim _{H} \varphi_{F}$ exists. Observe also that if $\left\langle F, \varphi_{F}\right\rangle$ is a labeled pair, $H \subseteq$ ess $G \subseteq$ ess $F$ and $\lim _{G} \varphi_{F}=r$, then also $\lim _{H} \varphi_{F}=r$. We may surely leave these two easy exercises to the reader.

One notion more: Given two labeled pairs $\left\langle F(0), \varphi_{F(0)}\right\rangle$ and $\left\langle F(1), \varphi_{F(1)}\right\rangle$, call them incompatible if for every $G \in \mathcal{G}$ that satisfies $G \subset^{\text {ess }} F(0)$ and $G \subset^{\text {ess }} F(1)$ simultaneously, at least one from $\lim _{G} \varphi_{F(0)}$ and $\lim _{G} \varphi_{F(1)}$ exists and equals 0 .

Suppose that $\left\langle F(0), \varphi_{F(0)}\right\rangle$ and $\left\langle F(1), \varphi_{F(1)}\right\rangle$ are two incompatible labeled pairs, $G(0) \subseteq$ ess $F(0), G(1) \subseteq{ }^{\text {ess }} F(1)$ and both limits $\lim _{G(0)} \varphi_{F(0)}, \lim _{G(1)} \varphi_{F(1)}$ exist and are positive. Then $G(0) \perp G(1)$. Indeed, if not, then $G(0) \| G(1)$ and there is a discrete family of cozero sets, say $H$, satisfying $H \subset \subset^{\text {ess }} G(0)$ and $H \subset \subset^{\text {ess }} G(1)$. Then $H \subset C^{\text {ess }} F(0)$ and $H \subset \subset^{\text {ess }} F(1)$, yet both limits $\lim _{H} \varphi_{F(0)}$ and $\lim _{H} \varphi_{F(1)}$ are positive. But this contradicts the incompatibility of $\left\langle F(0), \varphi_{F(0)}\right\rangle$ and $\left\langle F(1), \varphi_{F(1)}\right\rangle$.

We are ready to continue the proof. Let $A \in \mathcal{A}_{n}$. Fix a maximal pairwise incompatible family $\mathcal{C}(A)$ such that for each $\left\langle F, \varphi_{F}\right\rangle \in \mathcal{C}(A), F \subset^{\text {ess }} A$. For each $\left\langle F, \varphi_{F}\right\rangle \in \mathcal{C}(A)$, fix a maximal essentially disjoint family $\mathcal{E}(F)$ such that for every 
$G \in \mathcal{E}(F), G \subset \subset^{\text {ess }} F$ and there exists a positive $\operatorname{limit}_{\lim } \varphi_{F}$. Then let $\mathcal{B}(A)=$ $\bigcup\left\{\mathcal{E}(F):\left\langle F, \varphi_{F}\right\rangle \in \mathcal{C}(A)\right\}$ and $\mathcal{A}_{n+1}=\bigcup\left\{\mathcal{B}(A): A \in \mathcal{A}_{n}\right\}$.

We need to verify that $\mathcal{B}(A)$ is a maximal essentially disjoint family of elements satisfying (i), (ii) and (iii) from the proof of Lemma 2.2. Suppose to the contrary that there is some $G \in \mathcal{G}$ satisfying (i), (ii) and (iii) from the proof of Lemma 2.2 and such that $G \perp H$ whenever $H \in \mathcal{B}(A)$. Then $G$ belongs to no $\mathcal{E}(F)$ with $\left\langle F, \varphi_{F}\right\rangle \in \mathcal{C}(A)$. Fix $\left\langle F, \varphi_{F}\right\rangle \in \mathcal{C}(A)$ for a moment and consider the real $r$ defined by $r=\inf \left\{\sup \left\{\varphi_{F}(x): x \in \bigcup G \backslash \bigcup G^{\prime}\right\}: G^{\prime} \in[G]^{<\omega}\right\}$. If $r>0$, then the maximality of $\mathcal{E}(F)$ implies the existence of some $H \in \mathcal{E}(F)$ that is not essentially disjoint with $G$. This, however, is impossible by the assumption $G \perp H$. So $r=0$. Since this holds for every $\left\langle F, \varphi_{F}\right\rangle \in \mathcal{C}(A)$, it is enough to equip $G$ with some label $\varphi_{G}$, and then the labeled pair $\left\langle G, \varphi_{G}\right\rangle$ witnesses that $\mathcal{C}(A)$ is not a maximal pairwise incompatible family. We have achieved a contradiction. Thus every $\mathcal{B}(A)$ is maximal, and consequently $\mathcal{A}_{n+1}$ is, too.

As before, let $\mathcal{D}(X)=\bigcup_{n \in \omega} \mathcal{A}_{n}$. Aiming for the complete regularity of the space $\Psi(\mathcal{D}(X))$, let us show first that any mapping belonging to

$$
\left\{\varphi_{F}:(\exists n \in \omega)\left(\exists A \in \mathcal{A}_{n}\right)\left\langle F, \varphi_{F}\right\rangle \in \mathcal{C}(A)\right\}
$$

continuously extends to $\Psi(\mathcal{D}(X))$.

Choose $n \in \omega, A \in \mathcal{A}_{n}$ and $\left\langle F, \varphi_{F}\right\rangle \in \mathcal{C}(A)$ arbitrarily. Next, fix an arbitrary $f \in \operatorname{Ult}(\mathcal{D}(X))$ and denote by $B$ the unique element of $\mathcal{A}_{n}$ that is a member of $f$. We need to consider three cases:

(1) $A \neq B$. Then there is a finite family $B^{\prime} \subseteq B$ such that $\bigcup A \cap\left(\bigcup B \backslash \bigcup B^{\prime}\right)=\emptyset$, because $A \perp B$. We also have $F \subset^{\text {ess }} A$. Since the mapping $\varphi_{F}$ is a label for $F$, $\varphi_{F}$ is constantly 0 outside $\bigcup F$, in particular, on $\bigcup B \backslash \bigcup B^{\prime}$. Define $\psi(f)=0$, $\psi(x)=\varphi_{F}(x)$ for $x \in X$. Then $\psi$ is a continuous mapping, defined on $X \cup\{f\}$, that extends the mapping $\varphi_{F}$.

$A=B$. Let $C \in \mathcal{A}_{n+1} \cap f$, and we have the remaining two cases:

(2) $A=B$ and $C \notin \mathcal{E}(F)$. Then there is some $\left\langle G, \varphi_{G}\right\rangle \in \mathcal{C}(A)$ such that $C \in \mathcal{E}(G)$ and $F, G$ are incompatible. According to our choice of $\mathcal{E}(G)$, the limit $\lim _{C} \varphi_{G}$ exists and is equal to a positive real number.

From the incompatibility of $\left\langle G, \varphi_{G}\right\rangle$ with $\left\langle F, \varphi_{F}\right\rangle$, either $\lim _{C} \varphi_{F}$ does not exist or it is 0 . But if $\lim _{C} \varphi_{F}$ does not exist, then there are some $D \subset \subset^{\text {ess }} C$ and a positive real number $r$ such that $\lim _{D} \varphi_{F}=r$. Then the maximality of $\mathcal{E}(F)$ implies that the family $D$ and hence also $C$ is essentially compatible with some member from $\mathcal{E}(F)$, which contradicts the essential disjointness of $\mathcal{A}_{n+1}$. Therefore $\lim _{C} \varphi_{F}=0$.

Define $\psi(f)=0, \psi(x)=\varphi_{F}(x)$ for $x \in X$ in this case. Given a real $\varepsilon>0$, the equality $\inf \left\{\sup \left\{\varphi_{F}(x): x \in \bigcup C \backslash \bigcup C^{\prime}\right\}: C^{\prime} \in[C]^{<\omega}\right\}=0$ yields a finite $C^{\prime} \subseteq C$ with $\sup \left\{\varphi_{F}(x): x \in \bigcup C \backslash \bigcup C^{\prime}\right\}<\varepsilon$. Taking into account that $\varphi_{F} \geq 0$ everywhere on $X$, this shows the continuity of $\psi$ on $X \cup\{f\}$.

(3) $A=B$ and $C \in \mathcal{E}(F)$. Then there is a positive real $r$ with $\lim _{C} \varphi_{F}=$ $r$. Define $\psi(f)=r, \psi(x)=\varphi_{F}(x)$ for $x \in X$. If $\varepsilon>0$, there is some finite subfamily $C^{\prime} \subseteq C$ such that $\sup \left\{\varphi_{F}(x): x \in \bigcup C \backslash \bigcup C^{\prime}\right\}<r+\varepsilon$, and another one $C^{\prime \prime} \in[C]^{<\omega}$ such that $\inf \left\{\varphi_{F}(x): x \in \bigcup C \backslash \bigcup C^{\prime \prime}\right\}>r-\varepsilon$. So for every $x \in O\left(f, C, C^{\prime} \cup C^{\prime \prime}\right) \cap(X \cup\{f\})$ we have $\psi(x) \in(r-\varepsilon, r+\varepsilon)$.

Since the mapping $\varphi_{F}$ has a continuous extension on each subspace $X \cup\{f\}, f \in$ $\Psi(\mathcal{D}(X)) \backslash X$, there is a continuous extension of $\varphi_{F}$ on the whole space $\Psi(\mathcal{D}(X))$. Let us use the symbol $\varphi_{F}$ also for the extended mapping. 
To conclude the proof of the complete regularity, let $f \in \operatorname{Ult}(\mathcal{D}(X))$ and let $O\left(f, A, A^{\prime}\right)$ be an open neighborhood of it, where $A \in \mathcal{A}_{n}$ and $A^{\prime}$ is a finite subfamily of $A$. We have a unique $C \in \mathcal{A}_{n+1} \cap f$. Then $C \subseteq{ }^{\text {ess }} A$, and hence there is some $F \subseteq$ ess $A,\left\langle F, \varphi_{F}\right\rangle \in \mathcal{C}(A)$, such that $C \in \mathcal{E}(F)$. Consider the mapping $\varphi_{F}$. We already know from case (3) above that $\varphi_{F}(f)=r>0$. By case (1) of our previous discussion, if $g \in \widehat{B}$ for $B \in \mathcal{A}_{n}, B \neq A$, then $\varphi_{F}(g)=0$. Also, $F \subseteq \subseteq^{\text {ess }} A$ and $\varphi_{F}(x)=0$ for all $x \in X \backslash \bigcup F$. So $\varphi_{F}$ is a continuous mapping with a positive value in $f$ and zero outside $O(f, A, \emptyset)$. Since $A$ is a discrete collection in $X$ and $\bigcup A^{\prime}$ is a relatively pseudocompact subset of $X$, the existence of a continuous mapping, which is positive at $f$ and zero outside $O\left(f, A, A^{\prime}\right)$, trivially follows.

Recall that a topological space $X$ is densely countably compact if $X$ has a dense subset $D$ that is relatively countably compact in $X$, i.e., every infinite subset of $D$, if not closed in $D$, is also not closed in $X$.

Our next theorem is a strengthening of $[\mathrm{W}$, Theorem 3.1].

Theorem 2.8. There is a Moore, connected, pseudocompact Tychonoff space without a dense relatively countably compact subset.

Proof. Our aim is to find the space in question as $\Psi(\mathcal{D}(\mathbb{R}))$. Apply Theorem 2.7 to $\mathbb{R}$. Then the resulting space is Moore, connected, pseudocompact and Tychonoff.

It turns out that the choice of the first maximal essentially disjoint family $\mathcal{A}_{0}$ can kill all possible candidates for a dense relatively countably compact subset. Let us observe:

Pick an arbitrary $F \in \mathcal{A}_{0}$ and an arbitrary $G \in \mathcal{A}_{1}$. Then there is a cofinite $G^{\prime} \subseteq G$ such that $\bigcup G^{\prime} \cap \bigcup_{U \in F} b d U=\emptyset$, because either $G \subset^{\text {ess }} F$ or $G \subset \subset^{\text {ess }} F^{\prime}$ with $F^{\prime} \in \mathcal{A}_{0}, F^{\prime} \perp F$. Therefore $\{b d U: U \in F\}$ is a discrete family in $\Psi(\mathcal{D}(\mathbb{R}))$, with all members nonempty, because the real line $\mathbb{R}$ is connected.

For every $n \in \omega$, choose a pairwise disjoint family consisting of $2^{n}$ closed nondegenerate subintervals of an interval $(n, n+1)$ and enumerate it as $\left\{J_{s}: s \in{ }^{n} 2\right\}$. Next, let $\left\{D_{f}: f \in{ }^{\omega} 2\right\}$ be the list of all countable dense subsets of $\mathbb{R}$.

For $f \in \omega^{\omega} 2$ and $n \in \omega$, choose an open nonempty subinterval $U(f, n) \subseteq J_{f i n}$ with endpoints in $D_{f}$. This is always possible, since $D_{f}$ is dense. Let $\mathcal{A}_{0}$ be an arbitrary maximal essentially disjoint family, $\mathcal{A}_{0} \supseteq\left\{\{U(f, n): n \in \omega\}: f \in{ }^{\omega} 2\right\}$.

It should be clear now that after such a start in the proof of Theorem 2.7, the space $\Psi(\mathcal{D}(\mathbb{R}))$ cannot have a dense relatively countably compact subset.

One remark should be added here. In Theorem 2.8, we meet with a case when the remainder $\Psi(\mathcal{D}(\mathbb{R})) \backslash \mathbb{R}$ with the subspace topology is homeomorphic to the generalized Baire space $\mathfrak{c}^{\omega}$. The Darboux property of continuous real functions guarantees that.

We conclude the paper with a theorem, mentioned in [T] and attributed by I. J. Tree to P. J. Nyikos. As it turned out after some e-mailing, the theorem still waits for a publication.

Theorem 2.9 (P. J. Nyikos, unpublished). Let X be a Tychonoff locally pseudocompact Moore space. The following are equivalent: (i) $X$ is separable, (ii) $X$ can be densely embedded in a pseudocompact Moore space, (iii) X can be densely embedded in a feebly compact Moore space.

Proof. (i) implies (ii): Theorem 2.7. (ii) implies (iii): Trivial. (iii) implies (i): Let $Y$ be the feebly compact extension of $X$. The space $X$ is locally pseudocompact, 
$Y$ is first countable, $X$ is dense in $Y$. So $X$ is open in $Y$. By [R1 Theorem 6], $Y$ is separable. Thus $X$ is separable.

\section{REFERENCES}

[B] Murray G. Bell, First countable pseudocompactifications, Topology Appl. 21 (1985), 159 166. MR 87b:54019

[E] Ryszard Engelking, General Topology, Heldermann Verlag, Berlin (1989). MR 91c:54001

[GJ] Leonard Gillman and Meyer Jerison, Rings of Continuous Functions, Van Nostrand, Princeton, NJ (1960). MR 22:6994

[R1] George M. Reed, On chain conditions in Moore spaces, General Topology and Appl. 4 (1974), 255 - 267. MR 49:9815

[R2] George M. Reed, On subspaces of separable first countable $T_{2}$-spaces, Fund. Math. 91 (1976), 189 - 202. MR 54:13863

[R3] George M. Reed, Set-theoretic problems in Moore spaces, in: Open Problems in Topology, edited by J. van Mill and G. M. Reed, North-Holland, Amsterdam, 1990, 163 - 181. MR 92c:54001

[S] Robert M. Stephenson, Jr., Moore-closed and first countable feebly compact extension spaces, Topology Appl. 27 (1987), 11 - 28. MR 89g:54034

[TT] Toshiji Terada and Jun Terasawa, Maximal extensions of first-countable spaces, Proc. Amer. Math. Soc. 85 (1) (1982), 95 - 99. MR 84a:54042

[T] Ian J. Tree, Extending the discrete finite chain condition, Topology Appl. 52 (1993), 267 278. MR 95d:54018

[W] W. Stephen Watson, A connected pseudocompact space, Topology Appl. 57 (1994), 151 162. MR 95g:54001

Department of Computer Science and Mathematical Logic, Charles University, Malostranské nám. 25, 11000 Praha 1, Czech Republic

E-mail address: psimon@ms.mff.cuni.cz

Dipartimento di Matematica, Universitì di Trieste, Via Alfonso Valerio 12/1, I-34127 Trieste, Italy

E-mail address: tironi@univ.trieste.it 